\title{
Choice of antimuscarinic agents for overactive bladder in the older patient: focus on darifenacin
}

\author{
Bilal Chughtai' \\ Robert Levin ${ }^{2}$ \\ Elise De' \\ 'Albany Medical College, Division \\ of Urology; ${ }^{2}$ Albany College of Pharmacy \\ and Stratton VAMC, Albany, NY, USA
}

\begin{abstract}
Overactive bladder $(\mathrm{OAB})$ is a difficult condition to live with and is very costly to the community. $\mathrm{OAB}$ affects $16 \%$ of the adult population and rises with increasing age. We describe the necessary steps in evaluation and behavioral therapy prior to initiating medical therapy. There are several medications that have been used for the treatment of patients who suffer from $\mathrm{OAB}$. This manuscript discusses the popular agents used for $\mathrm{OAB}$, with a focus on the recent clinical trials on darifenacin.
\end{abstract}

Keywords: darifenacin, overactive bladder, urge incontinence, older patients, aging

\section{Introduction}

Overactive bladder $(\mathrm{OAB})$ is a problem characterized by urgency; a sudden desire to urinate that cannot be postponed. According to the International Continence Society (ICS) "urgency, with or without urge incontinence, usually with frequency and nocturia, can be described as the overactive bladder syndrome, urge syndrome, or urgency-frequency syndrome. These terms can be used if there is no proven infection or obvious pathology."'(Wein and Rovner 2002). It can be associated with frequency and nocturia, and it may occur with urinary incontinence. $\mathrm{OAB}$ affects approximately $16 \%$ of the adult population in the US and the prevalence increases with age (Wein and Rovner 2002; Beneton and De Parisot 2003).

OAB costs an estimated US $\$ 12$ billion annually in the US, with US\$9 billion incurred in the community (Basra and Kelleber 2007). The estimate includes both the direct costs of care, protective undergarments, and treatment as well as consequential costs, such as those resulting from urinary tract infections and falls due to urgency to get to the bathroom. There are intangible costs such as pain, suffering, and poor quality of life. The negative impacts on health, the ability to function, and quality of life have been well-documented. The elderly with $\mathrm{OAB}$ and subsequent incontinence are more likely to be admitted to nursing homes. Thom et al (1997) found at least a 2 -fold increase in risk of admission to a nursing facility for patients with incontinence. Urinary incontinence can lead to anxiety, negative self-image, and isolation (Basra and Kelleber 2007). OAB places individuals at risk for other forms of morbidity. Problems associated with $\mathrm{OAB}$ include skin ulceration and urinary tract infections. Nocturia, waking up more than one time per night to void, is common with $\mathrm{OAB}$ and is among the most bothersome of lower urinary tract symptoms (Ohelke 2005). In addition to sleep interruption and resulting fatigue, patients with nocturia may be more likely to suffer from falls and fractures, which are associated with high morbidity in elderly patients ( $33 \%$ of patients do not survive beyond 1 year after a hip fracture) (Reeves et al 2006).

The immediate consequences and co-morbidities of $\mathrm{OAB}$ can have a negative influence on quality of life, particularly among the elderly. The effect of OAB becomes 
clearer with the National Overactive Bladder Evaluation (NOBLE) study (Coyne et al 2004). In this study, OAB was associated with much lower quality-of-life scores, higher scores on depression, and poorer sleep quality when compared to patient controls (Coyne et al 2004). Although OAB increases with age, it should not be considered a normal consequence of aging by patients or physicians. By using a systematic approach to diagnosing and evaluating OAB followed by instituting appropriate therapy, care providers can improve the health and quality of life of older patients with OAB.

\section{Normal bladder physiology}

Bladder function represents an interplay between the urinary tract and the nervous system. There are two main phases, filling and emptying. The normal bladder stores urine at a low pressure that remains lower than urethral resistance. Micturition is hallmarked by a decrease in urethral resistance and contractions of the detrusor muscle with resultant emptying.

There is a complex interplay of neurotransmitters and receptors leading to normal bladder function. Acetylcholine, by acting on muscarinic receptors within the bladder, is the primary neurotransmitter in bladder contraction (Yamanishi et al 2001). Contraction occurs as a result of increased parasympathetic outflow and relaxation of sympathetic inhibition. There are 5 known muscarinic subtypes (M1 to M5) that are distributed throughout the human body (Table 1). The muscarinic receptor subtypes are located in smooth muscle (bladder), exocrine glands, the nervous system, and the heart. M2 receptors are the predominant subtype within the healthy detrusor muscle; activation of M2 receptors inhibits sympathetically mediated detrusor relaxation. M2 receptors are also located in the heart and central nervous system (CNS). M3 receptors are responsible for detrusor smooth muscle contraction and also have an exocrine function in the salivary glands (Pontari et al 2004). The muscarinic receptor antagonists used to treat $\mathrm{OAB}$ may affect these receptors to varying levels. The use of muscarinic receptor antagonists

Table I Muscarinic receptors distribution (after Yamanishi et al 200 I)

\begin{tabular}{ll}
\hline Muscarinic receptor & Distribution \\
\hline MI & Brain (cortex, hippocampus), glands, \\
& sympathetic ganglia \\
M2 & Heart, hindbrain, smooth muscle \\
M3 & Smooth muscle, glands, brain \\
M4 & Brain (forebrain, striatum) \\
M5 & Brain (substantia nigra), eye \\
\hline
\end{tabular}

is associated with a spectrum of outcomes in both efficacy and side-effect profile.

\section{Evaluating patients with overactive bladder}

Patients with uncomplicated OAB can be managed by the primary care physician. The first step is to screen for a history of frequency, urgency, and incontinence. Unfortunately, many patients with $\mathrm{OAB}$ do not seek treatment because of embarrassment, the misconception that the problem is an inevitable consequence of aging, or that treatment options are limited. The history should focus on relevant medical, neurologic, and genitourinary symptoms that may lead to or worsen the symptoms of OAB. Neurologic conditions such as Alzheimer's or Parkinson's disease may affect cortical inhibitory function, which can cause or contribute to $\mathrm{OAB}$ (Stevens et al 2007). Conditions that affect the spinal cord (multiple sclerosis, disc herniation) may also disrupt normal micturition reflexes, resulting in OAB. Factors that affect the genitalia or the lower urinary tract, eg, bladder cancer, atrophic vaginitis, yeast infections, and cystitis, may lead to symptoms similar to $\mathrm{OAB}$. When dealing with symptoms of $\mathrm{OAB}$, it is imperative to identify causes that are easily reversed and appropriately manage these patients. In addition to a thorough history, assessment of fluid intake, voiding patterns, and real-time symptoms should be reviewed with the aid of a patient-completed voiding diary. In this exercise the patient logs intake of fluid, number of times voided, amount voided, and episodes of incontinence for at least one full day. It is important to asses for medical conditions that lead to increased production of urine during sleep, eg, diabetes mellitus, diabetes insipidus, sleep apnea, or congestive heart failure. Nocturnal polyuria is calculated as $>35 \%$ of daily urine production occurring during the sleep hours.

All medications should be reviewed for those that may affect urinary function, output, and so on. Diuretics, by increasing urinary output, clearly increase urinary frequency. Occasionally patients report night-time administration, a reversible cause of nocturnal polyuria. Various medications, including anticholinergics, narcotics, and calcium channel blockers, may decrease bladder contractility, leading to inability to completely empty the bladder and more frequent voiding. In addition, the use of sedatives may lead to deceased mobility and inability to get to the bathroom in time, leading to incontinence.

Physical examination should include the abdominal, genitourinary, rectal, and neurologic systems. It is important during the abdominal examination to make sure the bladder 
is not palpable. The genitourinary examination in women should include a pelvic examination to rule out an obstructing, kinking cystocele, atrophic vaginitis, and urethral diverticulum (palpable mass), and a rectal examination to rule out constipation, which can lead to OAB. In the male the size of the prostate and assessment for nodules is performed. Neurologic examination should include lumbar and sacral reflexes (anal wink, cremasteric, perianal sensation, bulbocavernosus reflex, anal sphincter tone) as well as mental status.

The properly collected and processed urinalysis is a mainstay in evaluation of OAB. Hematuria, glucosuria, proteinuria, and infection can be easily identified. If the urine dip is positive, it is imperative to send the specimen for microscopic examination. Hematuria is defined as $\geq 3$ red blood cells per high power field on two separate good quality specimens and should prompt comprehensive evaluation for neoplasm (renal mass protocol upper tract imaging, cystoscopy, and urine cytology) (Grossfield et al 2001a, b). Mensturation and vulvovaginal atrophy can contaminate the sample in women; recollection or catheterized specimens can be helpful.

The importance of obtaining a post void residual urine volume in the evaluation of OAB cannot be emphasized enough. This procedure can be performed by straight catheterization or bladder scanner in the office, or by ultrasound in radiology. Incomplete emptying is a common and potentially dangerous cause of OAB. It should be screened for in all new $\mathrm{OAB}$ patients. Incomplete emptying is more likely in those with cystocele or prostatic obstruction, neurogenic bladder, urinary tract infections, diabetes, and overflow (unawares) incontinence.

Serum creatinine is helpful in screening for compromised renal function. It is recommended (and can be complemented by renal ultrasound) in those with incomplete bladder emptying and suspected obstruction by pelvic organ prolapse, the prostate, or high-pressure neurogenic voiding dysfunction. Serum glucose should be obtained in those with polydypsia/polyuria, if diabetes is suspected.

\section{Behavioral intervention}

Intervention on reversible causes of OAB should be diagnosis-specific, for example reduction of a cystocele and removal of bladder tumor. Once these discreet diagnoses have been ruled out, treatment of uncomplicated $\mathrm{OAB}$ can begin. Behavioral intervention should always include patient education. Timed voiding in infrequent voiders or in patients with prior central nervous system injury (eg, stroke), decreased fluid consumption in those with poly- dypsia or night-time fluid intake, caffeine modification, and proper time of diuretic administration are all obvious places to start. Biofeedback pelvic floor muscle retraining can be used to relax any contributory pelvic floor muscle spasm and to educate patients on manipulation of bladder inhibitory reflexes. Unfortunately no peer-reviewed publication specifically exists for the use of biofeedback in OAB. However, a meta-analysis by Berghmans et al assessing the efficacy of biofeedback in urge urinary incontinence found that almost all studies had positive results in favor of biofeedback but better constructed studies were needed to really evaluate its efficacy (Berghmans et al 2000).

\section{Pharmacologic intervention}

There are many medications available for the management of uncomplicated OAB. Several agents previously used include propantheline, hyoscyamine, and imipramine. Propantheline bromide is a non-selective muscarinic receptor antagonist with low or varying bioavailability, with less frequent dosing and a larger side-effect burden (Nabi et al 2006). It is no longer applied for the treatment of OAB. Hyoscyamine sulfate, another muscarinic receptor antagonist, may be useful in patients with intermittent symptoms (Malone and Okano 1999). Its side effect profile includes prominent anticholinergic side effects. Imipramine, a tricyclic antidepressant, is not routinely recommended for the treatment of $\mathrm{OAB}$, but may be useful for stress incontinence or mixed urge-stress incontinence due to its effect on bladder neck tone (Wein 1998). Imipramine is associated with potentially serious CNS side effects and cardiotoxicity. More frequently prescribed agents for the treatment of $\mathrm{OAB}$ are oxybutynin, which has a mixed action, and tolterodine. Three additional drugs were approved by the US Food and Drug Administration in 2004 to treat the symptoms of $\mathrm{OAB}$ : trospium, which has been in use in Europe for the treatment of $\mathrm{OAB}$, darifenacin, and solifenacin (Lam and Hilas 2007).

Oxybutynin is one of the less selective muscarinic receptor antagonists (Schaefer 2007). Oxybutynin is available in 3 different formulations: immediate-release (taken twice daily), extended-release (taken once daily), and a transdermal patch (applied once every 3.5 days). Each has its own clinical niche. For instance, oxybutynin immediate release may be useful as a single dose in the evening for patients with nocturia who do not have troubling symptoms during the day.

Oxybutynin-ER has a wide dosing range from $5 \mathrm{mg}$ to $30 \mathrm{mg}$. This facilitates a dosing regimen that allows for a good balance between efficacy and tolerability. Oxybutynin is also available in a transdermal patch. Due to the fact 
that transdermal oxybutynin does not undergo first-pass metabolism in the liver, much lower concentrations of $N$-desethyloxybutynin, the active metabolite of oxybutynin, are noted. For this reason, in various clinical trials, transdermal oxybutynin has been shown to have a relatively low risk of side effects; most frequent was application-site reactions (Schaefer 2007). Even these may be reduced by the use of topical corticosteroid or antihistamine to the site.

Similar to oxybutynin, tolterodine is available in both short-acting and long-acting formulations. Both formulations have shown good efficacy for the symptoms of OAB in large studies (Choo et al 2008). The extended-release form is better-tolerated and more effective than the immediaterelease formulation (Choo et al 2008). In one randomized controlled study of extended-release tolterodine $4 \mathrm{mg} /$ day in patients with urge incontinence and urinary frequency, no differences were found for efficacy, tolerability, or safety between older and younger patients (Wefer et al 2001). In another trial, there was a small but statistically significant decrease in the efficacy of tolterodine with age (Michel et al 2002). In another study, The Overactive Bladder: Performance of Extended-Release Agents trial, oxybutynin-ER and extended-release tolterodine (tolterodine-ER) were compared. Reduction in the frequency of incontinence episodes was similar in the oxybutynin-ER and tolterodine-ER groups (Diokno et al 2003). Micturition frequency was more reduced in the oxybutynin-ER group than in the tolterodineER group. More importantly, a greater number of partients in the oxybutynin-ER group achieved complete continence (Diokno et al 2003). In a follow-up study using the same data set, central nervous system side effect profiles of oxybutynin-ER and tolterodine-ER were compared (Kay et al 2006). Adverse events occurred in $9.0 \%$ in the oxybutyninER group and $8.3 \%$ in the tolterodine-ER treatment groups $(p<0.8)$ (Kay et al 2006).

Certain risk profiles apply to all members of the anticholinergic class. All anticholinergic agents should be used with caution and specialty consultation in patients with narrow-angle glaucoma, decreased gastrointestinal motility, or clinically significant bladder outflow obstruction. Side effects of antimuscarinic agents can include changes in blood pressure, pulse rate or ECG when beginning treatment. Dizziness and blurred vision are occasionally reported as well. The most common side effect is dry mouth (Lam and Hilas 2007). Constipation and dyspepsia are also reported. Dizziness and somnolence are common CNS side effects. On the whole, side effects are transient and tolerable - although constipation is one of the common side effects, the use of laxatives and stool softeners is rarely necessary (Anon 2007). Adverse events or side effects infrequently lead to withdrawal of treatment.

Trospium chloride, which is a quaternary ammonium compound, has recently been approved by the FDA for the treatment of OAB (Staskin et al 2007). It was initially provided as a single $20 \mathrm{mg}$ dose for twice-daily use, and has more recently been approved in a $60 \mathrm{mg}$ once-daily formulation. Trospium efficacy has been shown to be equivalent to twice daily oxybutynin-IR with a lower incidence of dry mouth (Zinner et al 2004). Trospium also may have a lower risk of central nervous system side effects because of its low predilection to cross the blood-brain barrier (Guay 2003). It also has an added benefit of being metabolized by the kidneys rather than the cytochrome P450 system, thereby having fewer interactions with other medications (Guay 2003). It may be beneficial for patients who are elderly and/or receiving multiple medications.

More recent additions for the treatment of OAB are darifenacin and solifenacin, both of which are available in oncedaily formulations. Darifenacin is available in $7.5 \mathrm{mg} /$ day and $15 \mathrm{mg}$ /day formulations, with a recommended starting dose of $7.5 \mathrm{mg}$ daily. Solifenacin is started at $5 \mathrm{mg}$ once daily and dosing may be increased to $10 \mathrm{mg}$ once daily. These are M3-selective receptor antagonists which potentially may be more bladder-specific with reduced tendency for anticholinergic side effects. Agents that are M3-selective are associated with relatively high rates of constipation. Reported rates of $9.1 \%$ and $14.4 \%$ have been demonstrated for both solifenacin $10 \mathrm{mg}$ daily and darifenacin $7.5 \mathrm{mg}$ daily respectively (Anon 2005). Solifenacin should be used with caution in patients with reduced hepatic or renal function, and darifenacin is not recommended for use in patients with severe hepatic impairment. Darfenacin in the $7.5 \mathrm{mg}$ dose may be used with caution in patients with moderate hepatic impairment, and there are no dosing adjustments for patients with mild hepatic impairment. Darifenacin does not require dose adjustment for elderly patients, and both medications have been shown to have similar safety and efficacy between older and younger patients (Wagg et al 2006; Chancellor and Miguel 2007).

The literature is sparse on head to head trials of the newer agents. However, the STAR (solifenacin vs tolterodine multinational trial) trial compared $4 \mathrm{mg}$ of tolterodine to $5 \mathrm{mg}$ then $10 \mathrm{mg}$ of solfenacin (Chapple et al 2005a). At the $10 \mathrm{mg}$ dose of solifenacin, there was a $10 \%$ greater reduction in the number of reported incontinence episodes compared with the tolerodine group. Criticism of the study stems from the 
fact that FDA dosing allowed for an escalated $10 \mathrm{mg}$ dose of solifenacin, whereas tolterodine remained at $4 \mathrm{mg}$. In a more recently available subcut analysis of the data comparing tolterodine $4 \mathrm{mg}$ ER with solfenacin $5 \mathrm{mg}$, the authors found that within 4 weeks of solifenacin $5 \mathrm{mg}$ therapy, solifenacin $5 \mathrm{mg}$ was significantly better than tolterodine ER $4 \mathrm{mg}$ in improving incontinence episodes and reducing incontinence pad use. At the endpoint of the trial, there was a reduction in the number of incontinence episodes from 1.1 in the tolterodine arm and 1.6 in the solifenacin arm $(p=0.006)$ (Chapple et al 2005a, 2007a). Differences in efficacy continued for the duration of the study after the dose increase of solifenacin was allowed.

\section{Clinical trials of darifenacin}

Darifenacin has been the subject of several randomized clinical trials. Pooled data analysis from 3 studies enrolled over a thousand patients (85\% female; age range 19-88 years) with a minimum 6-month history of overactive bladder symptoms including urgency, frequency, and urge incontinence. All participants had a 2-week washout and a 2-week placebo run-in prior to the beginning of the trial. Patients were randomized to be given either 1) darifenacin $7.5 \mathrm{mg}$ or matched placebo or 2) darifenacin $15 \mathrm{mg}$ or matched placebo. Outcome data were collected by use of an electronic diary. Although there was a noted placebo response, both doses of darifenacin were superior to placebo in alleviating symptoms associated with OAB (Chapple et al 2005b). Darifenacin has also been compared with oxybutynin as well as a matched placebo. Seventy-six patients (93\% female) had detrusor overactivity verified with urodynamics with at least 4 urge incontinence episodes per week and a frequency of at least 8 micturitions per day. All patients underwent a 2 -week run-in period prior to 2 weeks of treatment in each arm. A paper diary was used to assess outcomes. Results revealed a comparable efficacy with oxybutynin, in comparison to the placebo arm with reduced a side effect profile (Chapple and Abrams 2005).

Another study looked at the onset of action of darifenacin. 561 patients (age range 19-88 years; 85\% female) with OAB symptoms were enrolled for at least 6 months (Haab et al 2004). After a 2 -week washout and a 2 -week placebo trial, participants were randomized to $3.75 \mathrm{mg}$ darifenacin, $7.5 \mathrm{mg}$ darifenacin, $15 \mathrm{mg}$ darifenacin or placebo. Onset of action was noted by 2 weeks, although increased benefit was seen up to 12 weeks in this study (Haab et al 2004).

Another parameter to measure the severity of urinary urgency includes warning time. This is the time between first sensation and time of micturition. This in theory represents the time available for the patient to find a toilet and could reduce social embarrassment. In a study by Chapple et al (2007b), 72 patients (51 female) with urinary urgency of more than 6 months duration and at least 4 urge episodes per day, received either darifenacin $30 \mathrm{mg}$ or placebo. All patients again had a 2 -week washout period and 2 weeks of treatment double blinded. Warning time was recorded before and after treatment using electronic event markers. The severity of the urgency was recorded on a Likert scale from 0 (no urgency) to 3 (severe urgency). After 2 weeks of treatment, the warning time increased in the patients taking darifenacin by $22.5 \%$ compared with a reduction of $18.9 \%$ in the placebo group (Chapple et al 2007b). This equated to a mean increase in warning time of $4.3 \mathrm{~min}$ in those taking darifenacin (Chapple et al 2007b). This may seem insignificantly small but especially in those at home or while in bed it provides time to find a toilet and avoid a socially embarrassing event of urge incontinence. Darifenacin's symptom improvement is supported by improvement in quality of life scores, as assessed by the King's Health Questionnaire (Nabi et al 2006).

In light of selectivity of muscarinic M3 receptors, darifenacin minimizes the risk of blockade of other muscarinic subtypes, eg, M1-mediated cognitive impairment. This is of particular importance for older patients who may be more susceptible to cognitive impairment and central nervous system effects. This patient population is at increased risk of cerebral exposure from increased permeability of the blood-brain barrier associated with advancing age, diabetes, Alzheimer's disease, or multiple sclerosis. There is a low incidence of cognitive side effects, which is especially beneficial for the elderly (Nabi et al 2006). In an analysis by Foote et al (2005), 317 patients aged 65 years or more were evaluated in one of three multicenter, randomized, double-blind, and placebo controlled studies. All patients had had symptoms of OAB for more than 6 months. If patients needed, they had a 2-week washout period and a 2-week drug-free/placebo run-in, prior to receiving once-daily darifenacin $7.5 \mathrm{mg}$ versus placebo, or darifenacin $15 \mathrm{mg}$ versus placebo. At 12 weeks, both doses of darifenacin were significantly superior along all symptoms of OAB assessed (Foote et al 2005). This analysis confirmed the efficacy in the elderly population. An important finding in this trial was the low incidence of nervous system effects (eg, somnolence, dizziness) during darifenacin therapy, similar to that with placebo in the present analysis of older patients, and also similar to that previously reported for the wider patient population. 
The favorable safety profile above is solidified by data showing that darifenacin is not associated with impairment of cognitive function, either in older or younger patients. This question has not been looked at specifically in any other antimuscarinic agent. Kay et al (2006) looked directly at cognitive function in 150 healthy subjects older than 60 years. Design included randomization to darifenacin, oxybutynin ER, or placebo in a multicenter, double-blind, double-dummy, parallel-group, 3-week study. Doses administered were oxybutynin ER $10 \mathrm{mg}$ once daily, increasing to $15 \mathrm{mg}$ then $20 \mathrm{mg}$ by week 3 and darifenacin $7.5 \mathrm{mg}$ daily in weeks 1 and 2, then $15 \mathrm{mg}$ in week 3 . The primary end point was accuracy on the Name-Face Association Test (delayed recall) at week 3. They found darifenacin had no significant effects on memory versus placebo, while oxybutynin ER caused significant memory deterioration, comparable to brain aging of 10 years. This study did have some limitations. These included 9 subjects who dropped from the darifenacin group, 6 from the oxybutynin group and 1 from the placebo group, which may have affected results. Also the study did use a higher dose of oxybutynin ER, up to $20 \mathrm{mg}$, while the darifenacin group only had 1 dose increase. There may have been differences in the group before starting treatment: the darifenacin group was slightly younger, has fewer females, and exhibited lower starting scores. Lastly, the duration examined was only 2 weeks.

The side effect profile of darifenacin is associated with a higher degree of constipation compared to oxybutynin, tolterodine, and trospium. Darifenacin at $7.5 \mathrm{mg}$ has $14.8 \%$ rate of constipation compared to $21.3 \%$ with the $15 \mathrm{mg}$ dose as per the product information insert. In addition, darifenicin has a $20.2 \%$ incidence of dry mouth at $7.5 \mathrm{mg}$ and $35 \%$ at the $15 \mathrm{mg}$ dose. These are important side effect profiles which may guide therapy especially in patients already suffering from pre-existing constipation or dry mouth.

\section{Discussion}

$\mathrm{OAB}$ has a significant impact on patient health and quality of life and is common in elderly patients. Once proper evaluation has ruled out complex causes of $\mathrm{OAB}$, many treatment options are available including behavioral and medical therapy. Ideally, therapy should begin with non-pharmacologic or behavioral therapy. Medical therapy should be considered if necessary. In the majority of the elderly population with $\mathrm{OAB}$, a muscarinic receptor antagonist should be able to provide substantial relief from the symptoms of $\mathrm{OAB}$, with a minimal side effect profile. With the variety of agents that are available today, and with continuing rigorous clinical trials, relief of symptoms can potentially be achieved for even the elderly patient population with OAB.

\section{Disclosures}

None of the authors has any conflicts of interest to disclose.

\section{References}

Anderson RU, et al. 2006. Effectiveness and tolerability of extended-release oxybutynin vs extended-release tolterodine in women with or without prior anticholinergic treatment for overactive bladder. Int Urogynecol J Pelvic Floor Dysfunct, 17:502-11.

Anon. 2005. Solifenacin and darifenacin for overactive bladder. Obstet Gynecol, 106:401-2.

Anon. 2007. Update on drugs for overactive bladder syndrome. Drug Ther Bull, 45:44-8.

Basra R, Kelleher C. 2007. Disease burden of overactive bladder: quality-of-life data assessed using ICI-recommended instruments. Pharmacoeconomics, 25:129-42.

Beneton C, De Parisot O. 2003. [The medical treatment of overactive bladder]. Neurochirurgie, 49:369-76.

Berghmans LC, et al. 2000. Conservative treatment of urge urinary incontinence in women: a systematic review of randomized clinical trials. BJU Int, 85:254-63.

Chancellor MB, Miguel F de. 2007. Treatment of overactive bladder: selective use of anticholinergic agents with low drug-drug interaction potential. Geriatrics, 62:15-24.

Chapple C, et al. 2005b. A pooled analysis of three phase III studies to investigate the efficacy, tolerability and safety of darifenacin, a muscarinic M3 selective receptor antagonist, in the treatment of overactive bladder. BJU Int, 95:993-1001.

Chapple C, et al. 2007b. Darifenacin treatment of patients $>$ or $=65$ years with overactive bladder: results of a randomized, controlled, 12-week trial. Curr Med Res Opin, 23:2347-58.

Chapple CR, et al. 2005a. A comparison of the efficacy and tolerability of solifenacin succinate and extended release tolterodine at treating overactive bladder syndrome: results of the STAR trial. Eur Urol, 48:464-70.

Chapple CR, et al. 2007a. Treatment outcomes in the STAR study: a subanalysis of solifenacin $5 \mathrm{mg}$ and tolterodine ER $4 \mathrm{mg}$. Eur Urol, 52:1195-203.

Chapple CR. Abrams P. 2005. Comparison of darifenacin and oxybutynin in patients with overactive bladder: assessment of ambulatory urodynamics and impact on salivary flow. Eur Urol, 48:102-9.

Choo MS, et al. 2008. Satisfaction with tolterodine: assessing symptomspecific patient-reported goal achievement in the treatment of overactive bladder in female patients (STARGATE study). Int J Clin Pract, 62:191-6.

Coyne KS, et al. 2004. The impact of urinary urgency and frequency on health-related quality of life in overactive bladder: results from a national community survey. Value Health, 7:455-63.

Diokno AC, et al. 2003. Prospective, randomized, double-blind study of the efficacy and tolerability of the extended-release formulations of oxybutynin and tolterodine for overactive bladder: results of the OPERA trial. Mayo Clin Proc, 78:687-95.

Foote J, et al. 2005. Treatment of overactive bladder in the older patient: pooled analysis of three phase III studies of darifenacin, an M3 selective receptor antagonist. Eur Urol, 48:471-7.

Grossfeld GD, et al. 2001a. Evaluation of asymptomatic microscopic hematuria in adults: the American Urological Association best practice policy-part II: patient evaluation, cytology, voided markers, imaging, cystoscopy, nephrology evaluation, and follow-up. Urology, 57:604-10. 
Grossfeld GD, et al. 2001b. Evaluation of asymptomatic microscopic hematuria in adults: the American Urological Association best practice policy-part I: definition, detection, prevalence, and etiology. Urology, 57:599-603.

Guay DR. 2003. Clinical pharmacokinetics of drugs used to treat urge incontinence. Clin Pharmacokinet, 42:1243-85.

Haab F, et al. 2004. Darifenacin, an M3 selective receptor antagonist, is an effective and well-tolerated once-daily treatment for overactive bladder. Eur Urol, 45:420-9; discussion 429.

Kay G, et al. 2006. Differential effects of the antimuscarinic agents darifenacin and oxybutynin ER on memory in older subjects. Eur Urol, 50:317-26.

Lam S, Hilas O. 2007. Pharmacologic management of overactive bladder. Clin Interv Aging, 2:337-45.

Malone DC, Okano GJ. 1999. Treatment of urge incontinence in Veterans Affairs medical centers. Clin Ther, 21:867-77.

Michel MC, et al. 2002. Does gender or age affect the efficacy and safety of tolterodine? J Urol, 168:1027-31.

Nabi G, et al. 2006. Anticholinergic drugs versus placebo for overactive bladder syndrome in adults. Cochrane Database Syst Rev, (4): CD003781.

Oehlke KJ. 2005. New treatment options for overactive bladder. $S D J$ Med, 58: 225-6.

Pontari MA, et al. 2004. The M2 muscarinic receptor mediates in vitro bladder contractions from patients with neurogenic bladder dysfunction. Am J Physiol Regul Integr Comp Physiol, 286:R874-80.

Reeves P, et al. 2006. The current and future burden and cost of overactive bladder in five European countries. Eur Urol, 50:1050-7.
Schaefer W. 2007. Comparison of the efficacy, safety, and tolerability of propiverine and oxybutynin for the treatment of overactive bladder syndrome. Int J Urol, 14:670; author reply 670-1.

Staskin D, et al. 2007. Once daily trospium chloride is effective and well tolerated for the treatment of overactive bladder: results from a multicenter phase III trial. J Urol, 178:978-83; discussion 983-4.

Stevens LA, et al. 2007. Human idiopathic and neurogenic overactive bladders and the role of M2 muscarinic receptors in contraction. Eur Urol, 52:531-8.

Thom DH, et al. 1997. Medically recognized urinary incontinence and risks of hospitalization, nursing home admission and mortality. Age Ageing, 26:367-74.

Wagg A, et al. 2006. Efficacy and tolerability of solifenacin in elderly subjects with overactive bladder syndrome: a pooled analysis. $\mathrm{Am} \mathrm{J}$ Geriatr Pharmacother, 4:14-24.

Wefer J, et al. 2001. Tolterodine: an overview. World J Urol, 19:312-8.

Wein AJ, Rovner ES. 2002. Definition and epidemiology of overactive bladder. Urology, 60(5 Suppl 1):7-12; discussion 12.

Wein AJ. 1998. Pharmacologic options for the overactive bladder. Urology, 51(2A Suppl):43-7.

Yamanishi T, et al. 2001. Which muscarinic receptor is important in the bladder? World J Urol, 19:299-306.

Zinner N, et al. 2004. Trospium chloride improves overactive bladder symptoms: a multicenter phase III trial. J Urol, 171:2311-5, quiz 2435. 
